\title{
Caracterización fonética de las variedades regionales del español y propuesta de transcripción simplificada
}

\author{
Germán COLOMA \\ Universidad del CEMA, Buenos Aires \\ gcoloma@cema.edu.ar
}

\begin{abstract}
RESUMEN
El presente trabajo identifica cinco características fonéticas (seseo, yeísmo, aspiración del fonema /s/, rehilamiento del fonema $/ 3 / \mathrm{y}$ aspiración del fonema $/ \mathrm{x} /$ ) cuya presencia o ausencia sirve para distinguir entre diez variedades regionales entre las cuales se puede dividir el universo de hablantes de la lengua española. En el trabajo se muestra también que para transcribir fonéticamente un texto tomando nota de dichas características diferenciadoras, basta utilizar 27 símbolos fonéticos, que constituyen un repertorio fonológico ampliado que tiene en cuenta tanto los "fonemas controvertidos" como los "fonemas no controvertidos" del idioma español.
\end{abstract}

Palabras clave: características fonéticas, variedades regionales del español, fonemas controvertidos

[Recibido, mayo 2010; aprobado, diciembre 2010]

\section{Phonetic characterization of the regional varieties of Spanish and a proposal for a simplified transcription}

\begin{abstract}
This paper identifies five phonetic characteristics (seseo, yeismo, /s/-aspiration, rehilamiento, and / $\mathrm{x} /$-aspiration) whose presence or absence allows to distinguish among ten regional varieties in which the entire universe of Spanish speakers can be divided. We also show that, in order to phonetically transcribe a text, taking into account those distinguishing characteristics, it is enough to use 27 phonetic symbols, that constitute an extended phonological inventory that includes both the "controversial" and the "non-controversial" Spanish phonemes.
\end{abstract}

Keywords: phonetic characteristics, Spanish regional varieties, controversial phonemes

\section{Introducción}

El objetivo de este artículo es analizar las principales características fonéticas que diferencian a las distintas variedades regionales del idioma español, y elaborar una propuesta de transcripción simplificada que utilice un número reducido de símbolos pero que a la vez distinga entre las distintas variedades regionales identificadas. Para ello el trabajo se divide en una primera sección que describe los principales sonidos y fonemas que se utilizan para hablar la lengua española, una segunda sección que describe los principales sistemas fonológicos vigentes en las zonas en las que se habla el idioma español, una tercera sección en la cual aparece una propuesta de transcripción fonética simplificada, y una cuarta sección de conclusiones. 


\section{Sonidos y fonemas del idioma español}

Los sonidos que se utilizan para hablar el idioma español, al igual que los de la mayoría de las lenguas de origen europeo, se pueden clasificar de manera bastante tajante en vocales y consonantes. Las vocales son las emisiones de voz que no encuentran ningún obstáculo en su recorrido a lo largo del aparato fonador, en tanto que las consonantes son las emisiones de voz que encuentran algún obstáculo en dicho recorrido. En español, además, las sílabas precisan contar con al menos una vocal, no existiendo por lo tanto casos de sílabas puramente consonánticas.

Los sonidos vocálicos del español son esencialmente cinco, y se representan a través de los signos fonéticos [a], [e], [i], [o] y [u]. Dichos sonidos se diferencian entre sí por su punto de articulación, que puede ser más o menos abierto o cerrado, y más o menos anterior o posterior (ver cuadro 1). Cada uno de estos cinco sonidos tiene a su vez características adicionales que lo identifican. Los sonidos [a], [e] e [i], por ejemplo, son vocales no labializadas; en tanto que los sonidos [o] y [u] son vocales labializadas. Cada uno de ellos puede presentar ciertas variaciones en cuanto a su grado de apertura y posicionamiento, pero en todas las posiciones en las que aparecen dentro del idioma español mantienen sus características básicas y su capacidad de oponerse unos a otros ${ }^{1}$. Esto vale tanto para los casos de sílabas tónicas como átonas, y para los casos en los cuales los sonidos vocálicos aparecen formando diptongos o triptongos.

$\begin{array}{lccc}\text { Tipo de vocal } & \text { Anterior } & \text { Central } & \text { Posterior } \\ \text { Cerrada } & \mathrm{i} & & \mathrm{u} \\ \text { Media } & \mathrm{e} & & \mathrm{o}\end{array}$

Abierta

a

Cuadro 1: Sonidos vocálicos del español

En cuanto a los sonidos consonánticos del español, los mismos se diferencian entre sí según su punto de articulación y también según su modo de articulación. Según su punto de articulación, dichos sonidos pueden ser labiales, dentales, alveolares, palatales, velares o glotales. Según su modo de articulación, la principal distinción que puede hacerse entre ellos es la que los divide en consonantes obstruyentes y sonantes ${ }^{2}$. Dentro del primer grupo aparecen las consonantes oclusivas y fricativas, que a su vez

\footnotetext{
${ }^{1}$ Tradicionalmente, y en especial a partir del trabajo de Navarro Tomás (1918), se ha considerado que en español existían también otros sonidos vocálicos que constituían variaciones de las cinco vocales descriptas. Los estudios experimentales más recientes ponen sin embargo en duda la existencia de dichos sonidos, que de cualquier modo no tienen en español ningún valor fonológico relevante. Al respecto, véase Martínez y Fernández (2007), capítulo 4.

${ }^{2}$ Esta clasificación está tomada de la que aparece en Martínez y Fernández (2007). Para otra clasificación alternativa, véase Frías Conde (2001).
} 
se subdividen en sonoras y sordas. Dentro del segundo grupo aparecen las consonantes nasales, laterales, róticas y semivocales (ver cuadro 2).

\begin{tabular}{|c|c|c|c|c|c|c|}
\hline Tipo de sonido & Labial & Dental & Alveolar & Palatal & Velar & Glotal \\
\hline Oclusivo sordo & $\mathrm{p}$ & & $\mathrm{t}$ & $t \int$ & $\mathrm{k}$ & \\
\hline Oclusivo sonoro & $b$ & & d & $d_{3}$ & g & \\
\hline Fricativo sonoro & $\beta$ & ð & $\mathrm{z}$ & 3 & $\gamma$ & \\
\hline Fricativo sordo & $\mathrm{f}$ & $\theta$ & $\mathrm{s}$ & $\int$ & $\mathrm{x}$ & $\mathrm{h}$ \\
\hline Nasal & $\mathrm{m}$ & & $n$ & $\mathrm{n}$ & $\eta$ & \\
\hline Lateral & & & 1 & $\Lambda$ & & \\
\hline Rótico & & I & $\mathrm{I}$ & & & \\
\hline
\end{tabular}

Semivocal

Cuadro 2: Sonidos consonánticos del español

A efectos de interpretar los símbolos del cuadro 2, cabe aclarar algunas convenciones que se han utilizado. En primer lugar, en las series oclusivas y sonantes no se ha considerado procedente distinguir entre consonantes dentales y alveolares, por no ser dicha distinción relevante para estudiar los sonidos del idioma español. En segundo lugar, los sonidos palatales oclusivos representados en el cuadro $([\mathrm{t}]]$ y $\left.\left[\mathrm{d}_{3}\right]\right)$ son en rigor sonidos africados, es decir, presentan un elemento oclusivo seguido de un elemento fricativo. En tercer lugar, para el caso de las consonantes róticas, el sonido $[r]$ representa a una consonante vibrante simple, en tanto que el sonido $[\mathrm{r}]$ corresponde a una consonante vibrante múltiple y el sonido $[\mathrm{I}]$ es en cambio una consonante asibilada cuyas características la acercan al grupo de las consonantes fricativas. Las tres se articulan en el mismo punto (alveolar).

Los sonidos que aparecen en los cuadros 1 y 2 se utilizan en el idioma español para articular los distintos fonemas que constituyen el sistema fonológico de la lengua. En algunos casos, dichos fonemas tienen una única realización, es decir, se pronuncian utilizando un único sonido. En otros casos, los hablantes utilizan más de un sonido para articular un único fonema, apareciendo en dichas circunstancias una serie de alófonos de los fonemas en cuestión. Por último, existen también en español ciertas variaciones regionales, que hacen que hablantes de distintas regiones utilicen distintos sonidos para articular el mismo fonema o alguno de sus alófonos.

El número de fonemas del español es también distinto según la variedad regional que se analice. La mayoría de los textos sobre fonología española describen como variedad principal un tipo de lengua (que llamaremos "castellano tradicional") que cuenta con 24 fonemas distintos. La mayoría de los hablantes de español, sin embargo, utilizan variedades que sólo emplean 22 ó a lo sumo 23 fonemas.

Al igual que en el caso de los sonidos que los forman, los fonemas también pueden clasificarse en principio en fonemas vocálicos y consonánticos. Los fone- 
mas vocálicos del español son cinco: /a/, /e/, /i/, /o/ y /u/. Los fonemas /a/, /e/ y /o/ se articulan esencialmente a través de los sonidos vocálicos [a], [e] y [o]. Los fonemas $/ \mathrm{i} / \mathrm{y} / \mathrm{u} /$, en cambio, se articulan a través de los sonidos vocálicos [i] y [u], salvo en los casos en los cuales aparecen en los denominados "diptongos crecientes" (/ia/, /ie/, /io/, /iu/, /ua/, /ue/, /ui/ y /uo/). En tales casos, el fonema/i/ se articula a través del sonido semivocal palatal [j], y el fonema $/ \mathrm{u} / \mathrm{se}$ articula a través del sonido semivocal labiovelar $[\mathrm{w}]^{3}$.

En lo que se refiere a los fonemas puramente consonánticos, los 17 fonemas obligatorios del español pueden escribirse utilizando los siguientes símbolos: /b/, $/ \mathbf{d} /, / \mathbf{f} /, / \mathbf{g} /, / \mathbf{k} /, / \mathbf{l} /, / \mathbf{m} /, / \mathbf{n} /, / \mathbf{n} /, / \mathbf{p} /, / \mathbf{r} /, / \mathbf{r} /, / \mathbf{s} /, / \mathbf{t} /, / \mathbf{t} \mathbf{\int} /, / \mathbf{x} /$ y $/ \mathbf{z} /$. Existen además otros dos fonemas optativos $(/ \mathbf{\Lambda} /$ y $/ \mathbf{\theta} /)$, que aparecen en algunas variedades regionales del idioma y no en otras. En aquellas variedades en las cuales los mismos no se utilizan, se reemplaza al fonema $/ \delta /$ por el fonema $/ 3 /$ (yeísmo) y al fonema $/ \theta /$ por el fonema /s/ (seseo).

Varios de los fonemas mencionados se articulan a través de un único sonido. Tal es el caso de los fonemas $/ \mathrm{f} /, / \mathrm{k} /, / 1 /, / \mathrm{m} /, / \mathrm{n} /, / \mathrm{p} /, / \mathrm{f} /, / \mathrm{t} /, / \mathrm{K} / \mathrm{y} / \theta /$, cuyo sonido coincide esencialmente con el del símbolo fonético que los identifica. Hay otros fonemas, en cambio, que poseen alófonos, es decir, sonidos alternativos que se usan para articular el mismo fonema en diferentes posiciones. Así, los fonemas oclusivos sonoros $/ \mathrm{b} /, / \mathrm{d} / \mathrm{y} / \mathrm{g} /$, poseen alófonos fricativos (o aproximantes) que son los sonidos $[\beta],[ð]$ y $[\gamma]$, respectivamente. De hecho, dichos alófonos son más frecuentes que los propios sonidos oclusivos, ya que estos últimos se utilizan solamente cuando el fonema respectivo aparece en posición inicial de palabra o cuando sigue a una consonante nasal. Otro caso de un fonema consonántico que tiene un alófono de importancia es el del fonema $/ \mathrm{n} /$, que se articula a través del sonido [ $\mathrm{y}$ ] cuando precede a una consonante velar (o sea, cuando aparece delante de $/ \mathrm{g} /, / \mathrm{k} / \mathrm{o} / \mathrm{x} /$ ), y a través del sonido [n] en el resto de los $\operatorname{casos}^{4}$.

En cuanto a los fonemas consonánticos $/ \mathrm{r} /, / \mathrm{s} /, / \mathrm{t} / /, / \mathrm{x} / \mathrm{y} / 3 /$, los mismos se caracterizan por tener realizaciones que varían según la región geográfica que se esté analizando. Numerosos hablantes sudamericanos articulan el fonema $/ \mathrm{r} / \mathrm{de}$ manera asibilada (a través del sonido [ $\mathrm{x}]$ ), en vez de utilizar la articulación vibrante múltiple. Del mismo modo, algunos hablantes del sur de España y de ciertas regiones de

\footnotetext{
${ }^{3}$ Existe sin embargo un caso (el del diptongo /ui/) que puede pronunciarse tanto de modo creciente como decreciente. En el primero de tales casos su articulación utiliza la semivocal [w], en tanto que en el segundo utiliza la vocal [u]. Un ejemplo del primer caso es el diptongo que aparece en la palabra "cuis" [kwis], en tanto que un ejemplo del segundo caso es el diptongo que aparece en la palabra "muy" [mui].

${ }^{4}$ En rigor, en español los fonemas $/ \mathrm{m} /, / \mathrm{n} / \mathrm{y} / \mathrm{n} /$ pierden su capacidad de oponerse unos a otros cuando están en posición preconsonántica, y adoptan el punto de articulación de la consonante que sigue. Podría por lo tanto decirse que no sólo [y] sino que también [m] y [n] son en ciertos casos alófonos del fonema $/ \mathrm{n} /$.
} 
América articulan el fonema $/ \mathrm{t} /$ / como una consonante fricativa palatal sorda [j], en vez de utilizar la articulación oclusiva o africada. Por último, cabe mencionar que la articulación del fonema fricativo velar sordo $/ \mathrm{x} /$ se vuelve en algunas regiones semejante a la de un sonido desbucalizado glotal [h], fenómeno este que se designa comúnmente como "aspiración" del fonema / $\mathrm{x} /$.

La desbucalización o aspiración afecta también, y de manera más generaliza$\mathrm{da}$, al fonema /s/, en especial cuando el mismo aparece en posición final de sílaba y precediendo a una consonante. Es así que, para algunos hablantes del idioma español, el fonema /s/ se articula a través de una consonante sonora alveolar bucalizada $[\mathrm{z}]$ cuando precede a una consonante sonora (y a través de una consonante sorda alveolar bucalizada [s] en el resto de los casos), en tanto que para otros hablantes el alófono del fonema /s/ no es [z] sino [h]. Dicho alófono es especialmente utilizado cuando el fonema /s/ aparece en posición preconsonántica, si bien algunos hablantes lo utilizan también en posición intervocálica.

El fonema /3/, por último, es quizás el que más variaciones regionales presenta. En las descripciones que toman como base al castellano tradicional, dicho fonema se articula generalmente como una consonante aproximante o semivocal palatal [j], y tiene un alófono africado palatal [d3] cuando aparece detrás de una consonante nasal o lateral ${ }^{5}$. En algunas variedades del español, dicho alófono africado se transforma en la realización normal del fonema $/ 3 /$ en todas las posiciones, existiendo también variedades en las cuales el fonema en cuestión se pronuncia en todas las posiciones de manera fricativa sonora [3] o, inclusive, a través del sonido fricativo palatal sordo [j]. A estos fenómenos de africación o asibilación se los conoce de manera genérica con el nombre de "rehilamiento" del fonema /3/.

\section{Caracterización fonética de las variedades regionales}

El idioma español puede subdividirse en una serie de variedades o dialectos, basándose en los distintos fonemas que los hablantes utilizan y en las distintas realizaciones que emplean para articular dichos fonemas. Piñeros (2008), por ejemplo, distingue tres grandes geolectos en los que puede dividirse el mundo hispanohablante, a los que denomina "peninsular norteño" (que abarca el centro y norte de España), "terrabajense" (que abarca el sur de España y numerosas zonas de América) y "terraltense" (que abarca el resto de las zonas del continente americano en las cuales se habla español como primera lengua). Usando una división geográfica más detallada, Moreno Fernández y Otero (2006) distinguen ocho áreas en las que subdividen a la lengua española, que son el área mexicano-centroamericana, el

\footnotetext{
${ }^{5}$ Véase, por ejemplo, Martínez y Fernández (2007). Estos autores distinguen sin embargo el sonido [j], típico del alófono semivocal del fonema $/ \mathrm{i} /$, del sonido aproximante del fonema $/ 3 /$, al que representan con el símbolo [j]. Asimismo, para el alófono africado de dicho fonema, que se representa generalmente con el símbolo [d3], utilizan el símbolo [łj].
} 
área caribeña, el área andina, el área chilena, el área rioplatense, el área andaluza, el área canaria y el área castellana. Esta última clasificación encaja exactamente dentro de la anterior, ya que las variedades mexicano-centroamericana y andina serían de tipo terraltense, las variedades caribeña, chilena, rioplatense, andaluza y canaria serían de tipo terrabajense, y la variedad castellana sería de tipo peninsular norteño.

Si empleamos una caracterización estrictamente fonológica de las principales diferencias que aparecen entre las distintas variedades del idioma español, sin embargo, las zonas en las cuales corresponde dividir al mundo hispanohablante son un tanto diferentes de las reseñadas por estos autores. Esto se debe a que la principal división que puede hacerse desde este punto de vista es entre áreas que utilizan 24 fonemas (es decir, zonas no sesantes ni yeístas), áreas que utilizan 23 fonemas (zonas no seseantes pero yeístas, y zonas no yeístas pero seseantes) y áreas que utilizan 22 fonemas (zonas seseantes y yeístas). La única variedad con 24 fonemas corresponde a una zona minoritaria del español peninsular norteño a la que denominaremos "castellano tradicional". El resto de los hablantes de español peninsular norteño pertenecen en cambio a un área yeísta pero no seseante, a la que denominaremos "español peninsular estándar", por ser esta la variedad que actualmente se considera como norma del español dentro de la península ibérica ${ }^{6}$. Por su parte, los hablantes de español que utilizan variedades seseantes pero no yeístas se encuentran ubicados casi en su totalidad en zonas que pertenecen a América del Sur, y que en la clasificación de Moreno Fernández y Otero entran dentro del área andina o del área rioplatense.

En lo que respecta a las zonas que son al mismo tiempo seseantes y yeístas, la principal división que puede hacerse desde el punto de vista fonético es la que las divide entre áreas en las cuales el fonema /s/ es comúnmente aspirado o desbucalizado en posición preconsonántica y áreas en las cuales dicho fenómeno no se produce ( $\mathrm{y}$, en consecuencia, el fonema /s/ en posición preconsonántica se pronuncia como [s] o como [z], según esté seguido de consonante sorda o sonora). Esta es en rigor la principal característica fonética que divide las variedades terrabajenses de las variedades terraltenses. Dicha división afecta también a las zonas seseantes no yeístas, ya que en las mismas es posible distinguir un área terrabajense de aspiración de /s/ preconsonántica (que corresponde al Paraguay y a varias provincias argentinas que limitan con dicho país, y que identificaremos como "español paraguayo") y un área terraltense en la cual dicha aspiración no es frecuente (que corresponde a las zonas no yeístas de Colombia, Perú y Bolivia, y que identificaremos como "español andino tradicional").

\footnotetext{
${ }^{6} \mathrm{Al}$ respecto véase, por ejemplo, Frías Conde (2001).
} 
En la zona terraltense yeísta, por su parte, la principal subdivisión fonética adicional que puede llevarse a cabo tiene que ver con la presencia o ausencia del fenómeno de africación generalizada del fonema /3/. Este es un rasgo común del área que Moreno Fernández y Otero denominan "mexicano-centroamericana" (que abarca México, Guatemala, El Salvador, Nicaragua, Honduras y Costa Rica), y que no es en cambio frecuente en el área andina (Ecuador, Perú, Bolivia y parte de Colombia). Al dialecto terraltense que presenta africación de $/ 3 /$ en todas las posiciones lo llamaremos por lo tanto "español mexicano-centroamericano", en tanto que al dialecto terraltense yeísta que no presenta dicha africación lo llamaremos "español andino moderno".

La africación de $/ 3 /$ en todas las posiciones es un rasgo que también ha sido señalado por Piñeros (2008) como característico del español del sur de España (andaluz y canario), y sirve por lo tanto para diferenciar a dicha variedad de las variedades terrabajenses americanas (caribeño, chileno y rioplatense). Si asimilamos la africación del fonema / 3 / con el fenómeno de asibilación de dicho fonema (es decir, con el uso del sonido [3] o del sonido [J] como articulación preponderante en todas las posiciones), podemos incorporar ambos fenómenos dentro del concepto más amplio de rehilamiento (que nos permite diferenciar al español rioplatense de las variedades caribeña y chilena). El rehilamiento por asibililación es también característico del español paraguayo (que Moreno Fernández y Otero consideran incluido dentro del español rioplatense), cuya gran diferencia fonética con el español rioplatense propiamente dicho es, como ya hemos mencionado, la ausencia de yeísmo.

Un último elemento que sirve para diferenciar entre las variedades del español terrabajense es la presencia o ausencia de aspiración o desbucalización del fonema $/ \mathrm{x} /$. Este fenómeno, que hace que dicho fonema se articule a través del sonido [h] en vez del sonido [x], aparece en el español andaluz-canario y en el español caribeño (que es el que se habla en Cuba, República Dominicana, Puerto Rico, Panamá, Venezuela y parte de Colombia), pero está ausente en el español chileno y en el español rioplatense y paraguayo. Con él podemos entonces completar la caracterización fonética de las diez variedades regionales del idioma español que hemos identificado, que son el castellano tradicional (CT), el español peninsular estándar (PS), el español andino tradicional (AT), el español andino moderno (AM), el español mexicano-centroamericano (MC), el español paraguayo (PA), el español chileno $(\mathrm{CH})$, el español rioplatense (RP), el español caribeño $(\mathrm{CB})$ y el español andaluz-canario (AC). Dicha caracterización es la que aparece en el cuadro 3. 


$\begin{array}{lcccccccccc}\text { Característica } & \text { CT } & \text { PS } & \text { AT } & \text { AM } & \text { MC } & \text { PA } & \text { CH } & \text { RP } & \text { CB } & \text { AC } \\ \text { Seseo } & \text { No } & \text { No } & \text { Sí } & \text { Sí } & \text { Sí } & \text { Sí } & \text { Sí } & \text { Sí } & \text { Sí } & \text { Sí } \\ \text { Yeísmo } & \text { No } & \text { Sí } & \text { No } & \text { Sí } & \text { Sí } & \text { No } & \text { Sí } & \text { Sí } & \text { Sí } & \text { Sí } \\ \text { Aspriación de } / \mathrm{s} / & \text { No } & \text { No } & \text { No } & \text { No } & \text { No } & \text { Sí } & \text { Sí } & \text { Sí } & \text { Sí } & \text { Sí } \\ \text { Rehilamiento de } / 3 / & \text { No } & \text { No } & \text { No } & \text { No } & \text { Sí } & \text { Sí } & \text { No } & \text { Sí } & \text { No } & \text { Sí } \\ \text { Aspiración de } / \mathrm{x} / & \text { No } & \text { No } & \text { No } & \text { No } & \text { No } & \text { No } & \text { No } & \text { No } & \text { Sí } & \text { Sí }\end{array}$

Cuadro 3: Diferencias fonéticas entre las variedades regionales del español

Tal como puede observarse, ninguna de estas diez variedades regionales es idéntica a otra en las cinco características fonéticas que hemos descripto. El orden en el cual las mismas aparecen en el cuadro 3 va desde las variedades más conservadoras hasta las menos conservadoras, notándose que en un extremo aparece el castellano tradicional (caracterizado por no poseer ni seseo, ni yeísmo, ni aspiración del fonema /s/, ni rehilamiento del fonema $/ 3 /$, ni aspiración del fonema $/ \mathrm{x} /$ ) y en el otro aparece el español andaluz-canario (caracterizado por poseer todas estas características fonéticas). Otra particularidad del cuadro 3 que vale la pena señalar es que las primeras dos columnas se refieren a variedades que entran dentro de la categoría de "español peninsular norteño", en tanto que las tres columnas siguientes corresponden a variedades de español terraltense, y las últimas cinco corresponden a variedades de español terrabajense.

El cuadro 3 nos recuerda además que la única de las diez variedades descriptas que utiliza 24 fonemas es el castellano tradicional (que presenta al mismo tiempo ausencia de seseo y de yeísmo), en tanto que con 23 fonemas aparecen el español peninsular estándar (yeísta pero no seseante), y el español andino tradicional y el español paraguayo (seseantes pero no yeístas). El resto de las variedades reseñadas son al mismo tiempo yeístas y seseantes, diferenciándose entre sí básicamente por el tipo de articulación que utilizan para los fonemas $/ \mathrm{s} /, \mathrm{x} / \mathrm{y} / 3 /$.

El resto de las variaciones regionales que pueden presentar los fonemas y alófonos del idioma español no sirven en cambio como elementos que ayuden a diferenciar unas variedades de otras. La asibiliación del fonema $/ \mathrm{r} /$, por ejemplo, es utilizada por numerosos hablantes de español andino, paraguayo y rioplatense, pero no es una característica que identifique a dichos dialectos ni que aparezca generalizada en la gran mayoría de los hablantes de los $\operatorname{mismos}^{7}$. Lo mismo puede decirse de la desafricación del fonema $/ \mathrm{t} /$, propia de algunos hablantes de español caribeño, chileno y andaluz-canario, pero que tampoco aparece generalizada y en muchos casos es considerada como subestándar por los hablantes de esos dialectos.

\footnotetext{
${ }^{7}$ Salvo que utilicemos una definición dialectal más estrecha, que identifique -por ejemplo-a una variedad de español del noroeste argentino, que podría caracterizarse como un dialecto que comparte todas las características del español rioplatense más la asibilación del fonema /r/. Al respecto, véase Fontanella (2000).
} 
Una característica que sí podría servir como diferenciadora de las diferentes variedades del idioma español, y que es comúnmente mencionada en los manuales de fonética, es la diferente articulación del sonido [s] que se utiliza en los dialectos castellano tradicional y peninsular estándar respecto del resto de las variedades regionales (americanas y andaluz-canaria). En el primero de tales grupos, el sonido [s] se articula de modo ápicoalveolar (más asibilado), en tanto que en el segundo grupo la articulación es dorsoalveolar (menos asibilado). Esta diferencia, sin embargo, tiene una correlación perfecta con la distribución geográfica del seseo, que aparece en todas las regiones donde el sonido [s] es dorsoalveolar y no aparece en cambio en aquellas áreas en las cuales dicho sonido es pronunciado de manera ápicoalveolar.

\section{Propuesta de transcripción fonética simplificada}

Para transcribir fonéticamente un texto, las alternativas básicas que existen son dos: o bien se utiliza una transcripción fonética propiamente dicha, que intenta representar los distintos sonidos que se utilizan al hablar una lengua, o bien se utiliza una transcripción fonológica, que busca representar los fonemas que se usan en dicha lengua. Por definición, una transcripción fonética utiliza siempre más símbolos que una transcripción fonológica, dado que emplea signos distintos para los diferentes alófonos que tienen los fonemas de un idioma, y también busca distinguir entre las distintas variaciones regionales que puede tener el mismo fonema o el mismo alófono.

Como ejemplo de lo dicho basta señalar que, en el presente trabajo, hemos utilizado 35 símbolos para describir fonéticamente los sonidos del idioma español, en tanto que para representar sus fonemas hemos usado solamente 24 símbolos $^{8}$. Una pregunta relevante al respecto podría ser entonces cuál es el número mínimo de símbolos que se necesita para representar al mismo tiempo todas las características fonológicas del español y todas las variaciones de las mismas que resultan relevantes para distinguir entre sus variedades regionales. Los 24 símbolos usados para describir los fonemas castellanos, por ejemplo, son suficientes para distinguir al castellano tradicional del español peninsular estándar, y a ambos del español andaluz-canario, pero no sirven para distinguir entre los distintos dialectos que se utilizan en el español americano, salvo para la distinción relativamente poco significativa que puede hacerse entre los dialectos no yeístas (andino tradicional y paraguayo) y los dialectos yeístas (todos los demás). En el otro extremo, usar los

\footnotetext{
${ }^{8}$ De hecho, limitar el número de sonidos del idioma español a 35 resulta en cierto modo arbitrario, ya que cada uno de dichos sonidos tiene variaciones de un hablante a otro que pueden hacer que resulte necesario usar un número mayor de símbolos para llevar a cabo ciertos estudios. Martínez y Fernández (2007), por ejemplo, incluyen en su libro un cuadro que distingue 59 sonidos distintos dentro del idioma español.
} 
35 símbolos fonéticos resulta redundante para diferenciar los dialectos descriptos en el presente trabajo, salvo que se quiera ahondar en la distribución de los alófonos dentro del habla o se quiera identificar características particulares de ciertos grupos de hablantes dentro de una misma variedad regional ${ }^{9}$.

Una alternativa intermedia que vale la pena explorar para elaborar una propuesta de transcripción fonética simplificada que cumpla con los requisitos deseados es partir de los 24 símbolos que representan a los fonemas españoles y adicionar algunos otros que representen lo que Hualde (2004) denomina "fonemas controvertidos". En efecto, decir que el español tiene 22, 23 ó 24 fonemas según se trate de hablantes que posean o no seseo o yeísmo es una descripción aceptada por la mayoría de los estudiosos de la fonética española, pero no es de ningún modo la única forma posible de estructurar el sistema fonológico de la lengua castellana. Existen por ejemplo autores que consideran que $/ \mathrm{j} / \mathrm{y} / \mathrm{w} /$ son fonemas separados y no alófonos de los fonemas /i/ y /u/, otros autores que consideran a los fonemas /i/ y /3/ como si fueran uno solo (sobre todo si están describiendo una variedad de español que utiliza al sonido [j] como articulación principal del fonema/3/), e inclusive autores que consideran a $[r]$ y [r] como alófonos de un mismo fonema ${ }^{10}$. Todas estas diferencias en cuanto a puntos de vista generan una "lista ampliada" de fonemas (controvertidos y no controvertidos) para la cual se necesitan 26 símbolos (los 24 utilizados por nosotros, más $/ \mathrm{j} / \mathrm{y} / \mathrm{w} /$ ).

A esta lista de 26 símbolos corresponde agregar uno más, que tiene que ver con un sonido que en ciertas variedades de español no se utiliza, pero que en otras aparece como alófono del fonema /s/ o como articulación excluyente del fonema /x/. Dicho símbolo es el que corresponde a la consonante fricativa aspirada glotal [h], que en el español caribeño y andaluz-canario adquiere propiamente el estatus de fonema (en reemplazo de $/ \mathrm{x} /$ ), y en el español rioplatense, chileno y paraguayo es un alófono del fonema /s/ que mantiene su oposición con el fonema $/ \mathrm{x} /$ cuando éste aparece en posición preconsonántica ${ }^{11}$.

Los 27 símbolos sugeridos pueden entonces emplearse para representar la pronunciación del idioma español en sus diez variedades regionales descriptas, formando un repertorio en el cual están incluidos todos los "fonemas posibles" (controvertidos y

\footnotetext{
${ }^{9}$ Como pueden ser, por ejemplo, los fenómenos de asibilación del fonema /r/ en el español andino, de desafricación del fonema $/ \mathrm{t}$ / en el español chileno, o de ensordecimiento del fonema $/ 3 /$ en el español rioplatense.

${ }^{10}$ Para un análisis detallado de este tema, véase Hualde (2004).

${ }^{11}$ Considérese, por ejemplo, los sintagmas nominales "reloj moderno" [re-'lox mo-'der-no] y "veloz modernidad" [be-'loh mo-der-ni-'da(d)], en cuya comparación puede apreciarse como el fonema $/ \mathrm{x} / \mathrm{y}$ el fonema /s/ (a través de su alófono [h]) mantienen su distinción cuando ambos aparecen delante del fonema $/ \mathrm{m} /$. Dicha oposición desaparece en las variedades de español que aspiran tanto el fonema $/ \mathrm{s} /$ como el fonema $/ \mathrm{x} /$ (caribeño y andaluz-canario), en las cuales el sonido $[\mathrm{h}] \mathrm{se}$ convierte en la forma principal (y única) de articular el fonema /x/, que en dichos dialectos debería por lo tanto ser representado como $/ \mathrm{h} /$.
} 
no controvertidos), incluido el fonema $/ \mathrm{h} /{ }^{12}$. Lo que se obtiene con estos 27 símbolos es a la vez una "transcripción fonética simplificada" y una "transcripción fonológica ampliada", ya que para algunas variedades los mismos son fonemas propiamente dichos y para otras representan alófonos de otros fonemas.

Considérese, por ejemplo, el caso de los símbolos [i], [j] y [3]. En el español peninsular estándar podemos considerar que $/ \mathrm{i} / \mathrm{y} / \mathrm{j} /$ son fonemas, que [3] es un alófono de $/ \mathrm{j} /$, y que $\left[\mathrm{j}\right.$ ] es asimismo un alófono de $/ \mathrm{i} /{ }^{13}$. En las variedades que presentan rehilamiento del fonema $/ 3 /$, en cambio, los fonemas son $/ \mathrm{i} / \mathrm{y} / 3 /$, y [j] es un alófono de /i/. Contrástese por ejemplo la secuencia de palabras "fierro - hierro - yerro", que en español peninsular estándar es ['fje-ro - 'je-ro - 'je-ro], en español rioplatense es ['fje-ro - 'je-ro - 'ze-ro], y en algunas variedades regionales puede llegar a convertirse en ['fje-ro - 'ze-ro - 'ze-ro].

Para mostrar cómo nuestro sistema de 27 símbolos sirve para representar las cinco características diferenciadoras de las diez variedades regionales de español reseñadas en el presente trabajo, considérese ahora el sintagma nominal "zapatilla magistral", que tiene la particularidad de pronunciarse de manera diferente en cada una de dichas variedades. Las transcripciones propuestas para dicho conjunto de palabras son las siguientes:

Castellano tradicional:

Peninsular estándar:

Andino tradicional:

Andino moderno:

Mexicano-centroamericano:

Paraguayo:

Chileno:

Rioplatense:

Caribeño:

Andaluz-canario:
[ $\theta$ a-pa-'ti- $K$ a ma-xis-'tral]

[ $\theta$ a-pa-'ti-ja ma-xis-'tral]

[sa-pa-'ti-Ka ma-xis-'tral]

[sa-pa-'ti-ja ma-xis-'tr al]

[sa-pa-'ti-za ma-xis-'tral]

[sa-pa-'ti- $\Lambda$ a ma-xih-'tral]

[sa-pa-'ti-ja ma-xih-'tr al]

[sa-pa-'ti-za ma-xih-'tral]

[sa-pa-'ti-ja ma-hih-'tral]

[sa-pa-'ti-za ma-hih-'tr al]

Tal como puede observarse, en este ejemplo los sonidos que se modifican al pasar de una variedad a otra son los que se representan ortográficamente a través de "z", "ll", "g" y "s", y que en las distintas variedades del idioma español adoptan formas que en nuestro sistema de transcripción representamos a través de los sím-

\footnotetext{
${ }^{12}$ En el apéndice 1 hemos incluido la lista completa de los 27 símbolos, y cuáles de ellos se usan para transcribir fonéticamente cada una de las variedades regionales del idioma español.

${ }^{13}$ Nótese que esto es en rigor una simplificación, ya que el verdadero alófono de /j/ en el castellano tradicional es el sonido africado [d3] y no el sonido fricativo [3], y de hecho el alófono semivocal de /i/ tampoco es absolutamente idéntico al sonido principal del fonema /3/. Por eso es que autores como Martínez y Fernández (2007) sostienen la necesidad de utilizar el símbolo [j] para representar al fonema que nosotros denotamos como /3/, y el símbolo [j] para el alófono semivocal $\mathrm{de} / \mathrm{i} /$.
} 
bolos $[\theta],[\mathrm{s}],[\Lambda],[\mathrm{j}],[3],[\mathrm{x}] \mathrm{y}[\mathrm{h}]$. Dichos símbolos denotan verdaderos fonemas en algunas variedades de español, y en otras pueden no existir o ser alófonos de algún otro fonema. Usando todos ellos, sin embargo, obtenemos una caracterización que resulta adecuada para representar el repertorio fonológico de cualquiera de las variedades descriptas, y la presencia o ausencia de las cinco características que hemos señalado como diferenciadoras de dichas variedades (es decir, seseo, yeísmo, aspiración de /s/, rehilamiento de /z/y aspiración de /x/ $)^{14}$.

\section{Conclusiones}

De todo lo expuesto y analizado en el presente trabajo resulta posible extraer una serie de conclusiones generales sobre las características fonéticas que presentan las distintas variedades regionales del idioma castellano, y sobre la posibilidad de representarlas a través de un número relativamente reducido de símbolos fonéticos.

Una primera conclusión que puede extraerse es que, a efectos de describir las principales características y diferencias entre las regiones que hablan español, basta con identificar cinco características fonéticas (seseo, yeísmo, aspiración de $/ \mathrm{s} /$, rehilamiento de $/ 3 /$ y aspiración de $/ \mathrm{x} /$ ), cuya presencia o ausencia permite tipificar diez variedades regionales (castellano tradicional, peninsular estándar, andaluz-canario, mexicano-centroamericano, caribeño, andino tradicional, andino moderno, chileno, rioplatense y paraguayo) que difieren entre sí en al menos una de las características analizadas.

Dichas características fonéticas diferenciadoras pueden ser descritas a través de un repertorio de solamente 27 símbolos, que representan los 24 fonemas que como máximo tiene el idioma español, más 3 sonidos que pueden ser considerados como "fonemas controvertidos" (es decir, como sonidos que en algunas variedades regionales y según algunas definiciones pueden ser considerados también fonemas), que son [j], $[\mathrm{w}]$ y [h]. Con ello podemos elaborar un repertorio que sirve para llevar a cabo transcripciones fonéticas simplificadas que son al mismo tiempo "transcripciones fonológicas ampliadas" de cualquier posible texto escrito en español, haciendo aparecer en ellas todas las particularidades fonéticas diferenciadoras de las variedades regionales en lo que hace a la presencia o ausencia de las cinco características básicas identificadas en el presente trabajo.

\footnotetext{
${ }^{14}$ En el apéndice 2 hemos incluido adicionalmente transcripciones fonéticas de un texto muy conocido a las distintas variedades regionales del idioma español, a fin de mostrar sus similitudes y diferencias en un contexto más amplio.
} 


\section{Apéndice 1: Repertorio de símbolos propuestos para la transcripción fonética}

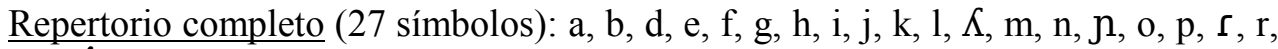
$\mathrm{s}, \mathrm{t}, \mathrm{t} f, \theta, \mathrm{u}, \mathrm{w}, \mathrm{x}, 3$.

Castellano tradicional (26 símbolos): a, b, d, e, f, g, i, j, k, l, $\Lambda, \mathrm{m}, \mathrm{n}, \mathrm{n}, \mathrm{o}, \mathrm{p}, \mathrm{r}, \mathrm{r}, \mathrm{s}$, $\mathrm{t}, \mathrm{t} f, \theta, \mathrm{u}, \mathrm{w}, \mathrm{x}, \mathrm{z}$ (no utiliza el símbolo "h").

Peninsular estándar (25 símbolos): a, b, d, e, f, g, i, j, k, l, m, n, n, o, p, r, r, s, t, t f, $\theta, \mathrm{u}, \mathrm{w}, \mathrm{x}, 3$ (no utiliza los símbolos " $\mathrm{h}$ " y " $\mathrm{K}$ ").

Andino tradicional (25 símbolos): a, b, d, e, f, g, i, j, k, 1, $\Lambda, \mathrm{m}, \mathrm{n}, \mathrm{n}, \mathrm{o}, \mathrm{p}, \mathrm{r}, \mathrm{r}, \mathrm{s}, \mathrm{t}$, t), u, w, x, 3 (no utiliza los símbolos " $h$ " y " $\theta$ ").

Andino moderno (24 símbolos): a, b, d, e, f, g, i, j, k, l, m, n, n, o, p, r, r, s, t, t f, u, w, $x, 3$ (no utiliza los símbolos " $h$ ", " $\Lambda$ " y " $\theta$ ").

Mexicano-centroamericano (24 símbolos): a, b, d, e, f, g, i, j, k, l, m, n, n, o, p, r, $\mathrm{r}, \mathrm{s}, \mathrm{t}, \mathrm{t}$ ), u, w, x, 3 (no utiliza los símbolos " $\mathrm{h}$ ", “ $\Lambda$ " y “ $\theta$ ”).

Paraguayo (26 símbolos): a, b, d, e, f, g, h, i, j, k, l, $\Lambda, \mathrm{m}, \mathrm{n}, \mathrm{j}, \mathrm{o}, \mathrm{p}, \mathrm{r}, \mathrm{r}, \mathrm{s}, \mathrm{t}, \mathrm{t}$, u, w, x, 3 (no utiliza el símbolo " $\theta$ ").

Chileno (25 símbolos): a, b, d, e, f, g, h, i, j, k, 1, m, n, n, o, p, r, r, s, t, t f, u, w, x, 3 (no utiliza los símbolos " $\Lambda$ " $y$ " $\theta$ ").

Rioplatense (25 símbolos): a, b, d, e, f, g, h, i, j, k, l, m, n, n, o, p, r, r, s, t, t f, u, w, $\mathrm{x,}, 3$ (no utiliza los símbolos " " $\mathrm{y}$ " $\theta$ ").

Caribeño (24 símbolos): a, b, d, e, f, g, h, i, j, k, l, m, n, n, o, p, r, r, s, t, t f, u, w, 3 (no utiliza los símbolos " $\Lambda$ " " $\theta$ " y " $x$ ").

Andaluz-canario (24 símbolos): a, b, d, e, f, g, h, i, j, k, 1, m, n, n, o, p, r, r, s, t, t f, u, w, 3 (no utiliza los símbolos " $\Lambda$ ", “ $\theta$ " $y$ “ $x ")$. 


\section{Apéndice 2: Ejemplos de transcripción fonética ${ }^{15}$}

Versión ortográfica del pasaje transcripto:El viento norte y el sol porfiaban sobre cuál de ellos era el más fuerte, cuando acertó a pasar un viajero envuelto en una ancha capa. El viento y el sol convinieron en que quien antes lograra obligar al viajero a quitarse la capa sería considerado más poderoso. El viento norte sopló con gran furia, pero cuanto más soplaba, más se arrebujaba en su capa el viajero. Por fin el viento norte abandonó la empresa. Entonces brilló el sol con ardor, e inmediatamente el viajero se despojó de su capa, por lo que el viento norte hubo de reconocer la superioridad del sol.

Transcripción fonética al castellano tradicional

el 'bje-nto 'nor-te jel 'sol por-'fja-ban so-bre 'kwal 'de-Kos 'e-ra el 'mas 'fwer-

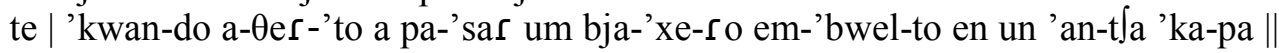
el 'bjen-to jel 'sol kom-bi-'nje-ron en ke kjen 'an-tes lo-'gra-ra o-bli-'gar al bja'xe-ro a ki-'tar-se la 'ka-pa se-'ri-a kon-si-de-'ra-do 'mas po-de-'ro-so \| el 'ßjen-to 'nor-te so-'plo kon 'gram 'fu-rja | 'pe-ro kwan-to 'ma so-'pla-ba | 'ma se a-re-bu-'xa-ba en su 'ka-pa el bja-'xe-ro || por 'fin el 'bjen-to 'nor-te aban-do-'no la em-'pre-sa || en-'ton- $\theta$ es bri-' $\hat{~}$ o el 'sol kon ar-'dor | e im-me-djata-'men-tel bja-'xe-ro se des-po-'xo de su 'ka-pa | por lo kel 'bjen-to 'nor-te 'ubo de re-ko-no-' $\theta$ er la su-pe-rjo-ri-'da del 'sol

\section{Transcripción fonética al español peninsular estándar}

el 'bje-nto 'nor-te jel 'sol por-'fja-ban so-bre 'kwal 'de-jos 'e-ra el 'mas 'fwerte | 'kwan-do a-Өer-'to a pa-'sar um bja-'xe-ro em-'bwel-to en un 'an-t $\int \mathrm{a}$ 'ka-pa || el 'bjen-to jel 'sol kom-bi-'nje-ron en ke kjen 'an-tes lo-'gra-ra o-bli-'gar al bja'xe-ro a ki-'tar-se la 'ka-pa se-'ri-a kon-si-de-'ra-do 'mas po-de-'ro-so el 'ßjen-to 'nor-te so-'plo kon 'gram 'fu-rja | 'pe-ro kwan-to 'ma so-'pla-ba | 'ma se a-re-bu-'xa-ba en su 'ka-pa el bja-'xe-ro || por 'fin el 'bjen-to 'nor-te aban-do-'no la em-'pre-sa || en-'ton-Өes bri-'jo el 'sol kon ar-'dor | e im-me-djata-'men-tel bja-'xe-ro se des-po-'xo de su 'ka-pa | por lo kel 'bjen-to 'nor-te 'ubo de re-ko-no-' $\theta$ er la su-pe-rjo-ri-'da del 'sol

\section{Transcripción fonética al español andino moderno}

\footnotetext{
${ }^{15}$ En este apéndice transcribiremos un pasaje de manera fonética a cada una de las diez variedades del español descriptas en el presente artículo, utilizando el repertorio de símbolos propuesto. El pasaje ha sido extraído de Jones y Dahl (1944), y aparece transcripto en numerosos trabajos sobre fonética española (por ejemplo, Quilis y Fernández, 1964; Martínez, Fernández y Carrera, 2003).

Para que el lector note la variación existente entre las distintas variedades descriptas, hemos resaltado las palabras que se pronuncian distinto en las diferentes variedades, conforme pasamos de una variedad a otra. Al final hemos incluido también una lista de las palabras con diferente pronunciación.
} 
el 'bje-nto 'nor-te jel 'sol por-'fja-ban so-bre 'kwal 'de-jos 'e-ra el 'mas 'fwerte | 'kwan-do a-ser-'to a pa-'sar um bja-'xe-ro em-'bwel-to en un 'an-t fa 'ka-pa || el 'bjen-to jel 'sol kom-bi-'nje-r on en ke kjen 'an-tes lo-'gr a-r a o-bli-'gar al bja'xe-ro a ki-'tar-se la 'ka-pa se-'ri-a kon-si-de-'ra-do 'mas po-de-'ro-so \| el 'ßjen-to 'nor-te so-'plo kon 'gram 'fu-rja | 'pe-ro kwan-to 'ma so-'pla-ba | 'ma se a-re-bu-'xa-ba en su 'ka-pa el bja-'xe-ro || por 'fin el 'bjen-to 'nor-te aban-do-'no la em-'pre-sa || en-'ton-ses bri-'jo el 'sol kon ar-'dor | e im-me-djata-'men-tel bja-'xe-ro se des-po-'xo de su 'ka-pa $\mid$ por lo kel 'bjen-to 'nor-te 'ubo de re-ko-no-'ser la su-pe-rjo-ri-'da del 'sol

Transcripción fonética al español mexicano-centroamericano

el 'bje-nto 'nor-te jel 'sol por-'fja-ban so-bre 'kwal 'de-zos 'e-r a el 'mas 'fwerte | 'kwan-do a-ser-'to a pa-'sar um bja-'xe-ro em-'bwel-to en un 'an-t fa 'ka-pa || el 'bjen-to jel 'sol kom-bi-'nje-r on en ke kjen 'an-tes lo-'gr a-ra o-bli-'gar al bja'xe-ro a ki-'tar-se la 'ka-pa se-'ri-a kon-si-de-'ra-do 'mas po-de-'ro-so \| el 'ßjen-to 'nor-te so-'plo kon 'gram 'fu-rja | 'pe-ro kwan-to 'ma so-'pla-ba | 'ma se a-re-bu-'xa-ba en su 'ka-pa el bja-'xe-ro || por 'fin el 'bjen-to 'nor-te aban-do-'no la em-'pre-sa || en-'ton-ses bri-'zo el 'sol kon ar-'dor | e im-me-djata-'men-tel bja-'xe-ro se des-po-'xo de su 'ka-pa $\mid$ por lo kel 'bjen-to 'nor-te 'ubo de re-ko-no-'ser la su-pe-rjo-ri-'da del 'sol

Transcripción fonética al español andino tradicional

el 'bje-nto 'nor-te jel 'sol por-'fja-ban so-bre 'kwal 'de-Kos 'e-ra el 'mas 'fwerte | 'kwan-do a-ser-'to a pa-'sar um bja-'xe-ro em-'bwel-to en un 'an-t fa 'ka-pa || el 'bjen-to jel 'sol kom-bi-'nje-r on en ke kjen 'an-tes lo-'gr a-ra o-bli-'gar al bja'xe-ro a ki-'tar-se la 'ka-pa se-'ri-a kon-si-de-'ra-do 'mas po-de-'ro-so \| el 'ßjen-to 'nor-te so-'plo kon 'gram 'fu-rja | 'pe-ro kwan-to 'ma so-'pla-ba | 'ma se a-re-bu-'xa-ba en su 'ka-pa el bja-'xe-ro || por 'fin el 'bjen-to 'nor-te aban-do-'no la em-'pre-sa || en-'ton-ses bri-'אo el 'sol kon ar-'dor | e im-me-djata-'men-tel bja-'xe-ro se des-po-'xo de su 'ka-pa | por lo kel 'bjen-to 'nor-te 'ubo de re-ko-no-'ser la su-pe-rjo-ri-'da del 'sol

\section{Transcripción fonética al español paraguayo}

el 'bje-nto 'nor-te jel 'sol por-'fja-ban so-bre 'kwal 'de-Kos 'e-ra el 'mah 'fwerte | 'kwan-do a-ser-'to a pa-'sar um bja-'xe-ro em-'bwel-to en un 'an-t fa 'ka-pa || el 'bjen-to jel 'sol kom-bi-'nje-ron en ke kjen 'an-teh lo-'gra-ra o-bli-'gar al bja'xe-ro a ki-'tar-se la 'ka-pa se-'ri-a kon-si-de-'ra-do 'mah po-de-'ro-so \| el 'bjen-to 'nor-te so-'plo kon 'gram 'fu-rja | 'pe-ro kwan-to 'ma so-'pla- $\beta a$ | 'ma se a-re-bu-'xa-ba en su 'ka-pa el bja-'xe-ro || por 'fin el 'bjen-to 'nor-te aban-do-'no la em-'pre-sa || en-'ton-seh bri-' $K o$ el 'sol kon ar-'dor | e im-me-djata-'men-tel bja-'xe-ro se deh-po-'xo de su 'ka-pa | por lo kel 'bjen-to 'nor-te 'ubo de re-ko-no-'ser la su-pe-rjo-ri-'da del 'sol 
Transcripción fonética al español rioplatense

el 'bje-nto 'nor -te jel 'sol por-'fja-ban so-bre 'kwal 'de-zos 'e-ra el 'mah 'fwerte | 'kwan-do a-ser-'to a pa-'sar um bja-'xe-ro em-'bwel-to en un 'an-t fa 'ka-pa || el 'bjen-to jel 'sol kom-bi-'nje-ron en ke kjen 'an-teh lo-'gra-ra o-bli-'gar al bja'xe-ro a ki-'tar-se la 'ka-pa se-'ri-a kon-si-de-'ra-do 'mah po-de-'ro-so \| el 'bjen-to 'nor-te so-'plo kon 'gram 'fu-rja | 'pe-ro kwan-to 'ma so-'pla- $\beta a$ | 'ma se a-re-bu-'xa-ba en su 'ka-pa el bja-'xe-ro || por 'fin el 'bjen-to 'nor-te aban-do-'no la em-'pre-sa || en-'ton-seh bri-' zo el 'sol kon ar-'dor | e im-me-djata-'men-tel bja-'xe-ro se deh-po-'xo de su 'ka-pa | por lo kel 'bjen-to 'nor-te 'ubo de re-ko-no-'ser la su-pe-rjo-ri-'da del 'sol

Transcripción fonética al español chileno

el 'bje-nto 'nor-te jel 'sol por-'fja-ban so-bre 'kwal 'de-jos 'e-ra el 'mah 'fwerte | 'kwan-do a-ser-'to a pa-'sar um bja-'xe-ro em-'bwel-to en un 'an-t fa 'ka-pa || el 'bjen-to jel 'sol kom-bi-'nje-ron en ke kjen 'an-teh lo-'gra-ra o-bli-'gar al bja'xe-ro a ki-'tar-se la 'ka-pa se-'ri-a kon-si-de-'ra-do 'mah po-de-'ro-so \| el 'bjen-to 'nor-te so-'plo kon 'gram 'fu-rja | 'pe-ro kwan-to 'ma so-'pla- $\beta a$ | 'ma se a-re-bu-'xa-ba en su 'ka-pa el bja-'xe-ro || por 'fin el 'bjen-to 'nor-te aban-do-'no la em-'pre-sa || en-'ton-seh bri-'jo el 'sol kon ar-'dor | e im-me-djata-'men-tel bja-'xe-ro se deh-po-'xo de su 'ka-pa | por lo kel 'bjen-to 'nor-te 'ubo de re-ko-no-'ser la su-pe-rjo-ri-'da del 'sol

Transcripción fonética al español caribeño

el 'bje-nto 'nor-te jel 'sol por-'fja-ban so-bre 'kwal 'de-jos 'e-ra el 'mah 'fwerte | 'kwan-do a-ser -'to a pa-'sar um bja-'he-ro em-'bwel-to en un 'an-t fa 'ka-pa || el 'bjen-to jel 'sol kom-bi-'nje-ron en ke kjen 'an-teh lo-'gra-ra o-bli-'gar al bja'he-ro a ki-'tar-se la 'ka-pa se-'ri-a kon-si-de-'ra-do 'mah po-de-'ro-so \| el 'bjen-to 'nor-te so-'plo kon 'gram 'fu-rja |'pe-ro kwan-to 'ma so-'pla-ba | 'ma se a-re-bu-'ha-ba en su 'ka-pa el bja-'he-ro \| por 'fin el 'bjen-to 'nor-te a-bando-'no la em-'pre-sa || en-'ton-seh bri-'jo el 'sol kon ar-'dor | e im-me-dja-ta'men-tel bja-'he-ro se deh-po-'ho de su 'ka-pa| por lo kel 'bjen-to 'nor-te 'u-bo de re-ko-no-'ser la su-pe-rjo-ri-'da del 'sol

Transcripción fonética al español andaluz-canario

el 'bje-nto 'nor-te jel 'sol por-'fja-ban so-bre 'kwal 'de-zos 'e-r a el 'mah 'fwerte | 'kwan-do a-ser-'to a pa-'sar um bja-'he-ro em-'bwel-to en un 'an-t fa 'ka-pa || el 'bjen-to jel 'sol kom-bi-'nje-ron en ke kjen 'an-teh lo-'gra-ra o-bli-'gar al bja'he-ro a ki-'tar-se la 'ka-pa se-'ri-a kon-si-de-'ra-do 'mah po-de-'ro-so \| el 'bjen-to 'nor-te so-'plo kon 'gram 'fu-rja |'pe-ro kwan-to 'ma so-'pla-ba|'ma se a-re-bu-'ha-ba en su 'ka-pa el bja-'he-ro \| por 'fin el 'bjen-to 'nor-te a-bando-'no la em-'pre-sa || en-'ton-seh bri-' 30 el 'sol kon ar-'dor | e im-me-dja-ta'men-tel bja-'he-ro se deh-po-'ho de su 'ka-pa | por lo kel 'bjen-to 'nor-te 'u-bo de re-ko-no-'ser la su-pe-r jo-r i-' da del 'sol 
Lista de palabras con distinta pronunciación

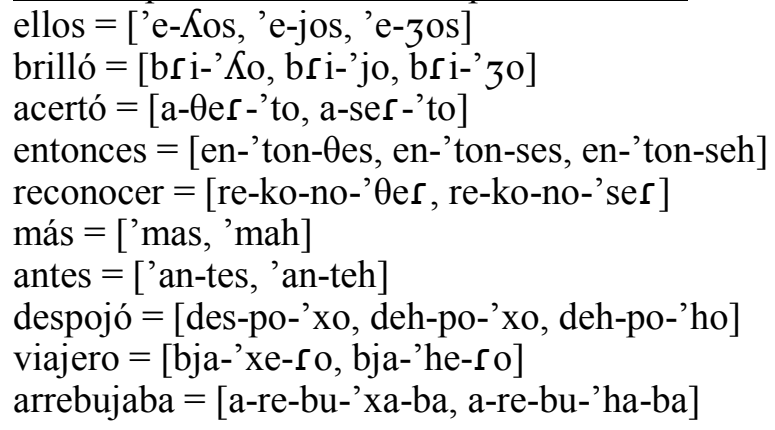

\section{Bibliografía}

FONTANELlA, Beatriz (2000). El español de la Argentina y sus variedades regionales. Buenos Aires, Edicial.

FRÍAS CONDE, Xavier (2001). "Introducción a la fonética y fonología del español"; Ianua, Revista Philologica Romanica, suplemento 4.

HuALDE, José (2004). "Quasi-Phonemic Contrasts in Spanish"; WCCFL 23 Proceedings, pp 1-25.

JONES, Daniel y DAHL, Ivar (1944). Fundamentos de escritura fonética según el sistema de la Asociación Fonética Internacional. Londres, University College.

MARTíneZ, Eugenio y FERNÁNDEZ, Ana (2007). Manual de fonética española. Barcelona, Ariel.

MARTínEZ, Eugenio; FernÁNDEZ, Ana y CARRERA, Josefina (2003). "Castilian Spanish"; Journal of the International Phonetic Association, vol 33, pp 255260.

Moreno FernándeZ, Francisco y OTERO, Jaime (2006). "Demografía de la lengua española", DT 03/06; Madrid, Instituto Complutense de Estudios Internacionales.

NAVARRO TOMÁs, Tomás (1918). Manual de pronunciación española. Madrid, Centro de Estudios Históricos.

PIÑEROS, Carlos (2008). Estructura de los sonidos del español. Nueva York, Pearson.

QUILIS, Antonio y FERNÁNDEZ, José (1964). Curso de fonética y fonología espanolas. Madrid, CSIC. 